\title{
Association of Wilms' tumor 1 gene single-nucleotide polymorphism rs16754 with colorectal cancer
}

\author{
SURASAK SANGKHATHAT ${ }^{1}$, WANWISA MANEECHAY ${ }^{2}$, WELAWEE CHAIYAPAN ${ }^{3}$, \\ SAMORNMAS KANNGERN ${ }^{4}$ and TEERANUT BOONPIPATTANAPONG ${ }^{2}$
}

\author{
${ }^{1}$ Department of Surgery, Tumor Biology Research Unit; ${ }^{2}$ Central Research Laboratory; ${ }^{3}$ Department of Biomedical Science, \\ Biomedical Science Research Program, Faculty of Medicine, Prince of Songkhla University, Songkhla 90110; \\ ${ }^{4}$ Department of Pathology, Bumrungrad International Hospital, Bangkok 10110, Thailand
}

Received March 10, 2015; Accepted August 14, 2015

DOI: $10.3892 / \mathrm{mco} .2015 .647$

\begin{abstract}
Colorectal cancer (CRC) is one of the leading causes of cancer-related mortality worldwide. Our recent study demonstrated that the expression of Wilms' tumor 1 gene (WT1) is associated with surgical outcome in CRC patients. The present study aimed to investigate the genetic association of the single-nucleotide polymorphism rs16754 in the WT1 gene with the occurrence of CRC, using an age-matched case-control study design. In addition, the correlation between genotype and $W T 1$ expression was investigated. Genomic DNA samples from 104 CRC cases, aged 15-65 years, and 208 healthy controls, were genotyped for rs16754 using the TaqMan genotyping method. The genotype distribution conformed to the Hardy-Weinberg equilibrium $(\mathrm{P}=0.80)$. The overall minor allele frequency (MAF) of rs16754 (allele A) was 0.33 . The MAF among CRC cases was significantly higher compared with that in controls $(0.39$ vs. 0.31 , respectively; $\mathrm{P}=0.03)$. The AA genotype was significantly associated with the disease (odds ratio $=2.51,95 \%$ confidence interval: $1.24-5.07, \mathrm{P}=0.01$ ). Cases with the AA genotype exhibited a significantly poorer 3 -year overall survival (60\%), compared with those with the GG or GA genotypes (80\%) (log-rank test, $\mathrm{P}<0.01$ ). Reverse transcription quantitative polymerase chain reaction analysis demonstrated that the expression of WTI in tumor tissues was higher compared with that in normal tissue; however, there were no significant differences in its expression among different genotypes. Therefore, rs16754 was found to be associated with the occurrence and prognosis of CRC in our subjects.
\end{abstract}

Correspondence to: Dr Surasak Sangkhathat, Department of Surgery, Tumor Biology Research Unit, Faculty of Medicine, Prince of Songkla University, 15 Karnjanavanich Road, Songkhla 90110, Thailand

E-mail: surasak.sa@psu.ac.th

Key words: colorectal cancer, rs16754, Wilms' tumor 1 gene

\section{Introduction}

Colorectal cancer (CRC) is among the leading causes of cancer-related mortality. In the United States, it was estimated that 142,570 individuals were diagnosed with CRC in 2010, of whom 51,370 succumbed to the disease (1). In Thailand, CRC is the third most common type of cancer in men and the fifth in women. Furthermore, CRC statistics from 4 regional registries in the country, which have been collecting data for $>20$ years, clearly demonstrated that the incidence rates of CRC are continuously increasing (2). Between 2001 and 2003, the estimated incidence rates of CRC in Thailand were 11.3/100,000 men and $7.9 / 100,000$ women (2), while in 2008 , the respective incidence rates were 14.7/100,000 and 11.8/100,000 (3). In Southern Thailand, where this study was conducted, the statistics between 2004 and 2007 reported CRC incidences of 12.6/100,000 for men and 9.2/100,000 for women (4).

Although surgery is the mainstay of treatment for early-stage $\mathrm{CRC}$, there is an increasing use of multidisciplinary treatment. As adjuvant treatments are considered based on individual patient risk, the identification of prognostic factors is crucial for risk stratification. Well-known factors affecting outcome in CRC patients are tumor invasion, nodal status, metastatic status and carcinoembryonic antigen level $(5,6)$.

Various histological parameters and biological markers have been investigated for possible associations with CRC. Biological markers, such as the DNA mismatch repair genes DCC, NM23-H1 and K-Ras have been verified as being able to predict disease relapse (7-10). The application of those molecular markers at the clinical level remains limited, however, due to limitations in their reproducibility and independency from other major prognosticators. Over several years, our research team has been focusing on the prognostic role of Wilms' tumor 1 gene (WTI) expression in CRC and have found that high WT1 expression is correlated with poor survival, regardless of tumor stage (11). These findings prompted us to investigate the genetic epidemiology of WT1 in the Southern Thailand population and its association with the occurrence, severity and prognosis of CRC.

$W T 1$ is among the biological markers that have been proven to act as oncogenes in various human cancers (12). WT1, located on chromosome 11p13, encodes a zinc finger transcription 
factor that plays an important role in cell growth and differentiation. In humans, WT1 is mainly expressed in the embryonic genitourinary system. Pathologically high WT1 expression has been reported in acute leukemic cells (13), lung cancer (14), breast cancer $(15)$ and CRC $(11,16)$. Immunotherapy against the WT1 antigen as an adjuvant biological treatment for cancer is currently actively investigated globally $(12,17)$.

The aim of this study was to investigate the single-nucleotide polymorphism (SNP) rs16754 of WT1 in CRC patients. This variant was previously extensively investigated in childhood hematological malignancies (18-20), but has never been investigated in CRC. This study was undertaken to determine whether rs16754 is associated with the occurrence of CRC, as well as its correlation with pathological parameters and clinical outcomes following surgery, particularly survival probability.

\section{Subjects and methods}

Patients and controls. Patients aged 15-65 years with histologically proven colorectal adenocarcinoma, who underwent definitive surgery at Songklanagarind Hospital (Hat Yai, Thailand) between January, 2006 and June, 2013, were asked to participate in this study. A cut-off of 65 years was selected to reduce interference caused by other carcinogenic factors associated with ageing. CRC patients with familial adenomatous polyposis coli syndrome were also excluded. Blood samples were collected under informed consent. Subjects who had no history of cancer were recruited from community-based volunteers residing in the Songkhla province to be used as controls. Age-matched controls were selected on an individual basis without prior knowledge of the WT1 rs16754 genotypes, with an age difference of $\leq 2$ years between each case and the matched controls. In order to increase the power of the study, the number of controls was twice that of the CRC cases.

The current clinical practice guideline for CRC patients, including preoperative investigation and postoperative follow-up in our institution, was published in our previous study (21). Briefly, adjuvant chemotherapy was considered for stage III colon cancer patients, and adjuvant chemoradiation was considered for stage II and III rectal cancer cases. Patients who received neoadjuvant treatment were not included in this study. All the patients were evaluated for $\geq 1$ year following surgery, or until death. Follow-up visits were scheduled at 1-month intervals during the first year following surgery, every 3 months during the second year, and every 6 months thereafter. Tumor staging was performed according to the sixth edition of the TNM staging system of the American Joint Committee on Cancer, version 5 (22). Our request for access to pathological samples and clinical records was approved by the Institutional Research Ethics Committee of Prince of Songkhla University (Songkhla, Thailand).

Extraction of DNA and genotyping of WT1 rs16754. Genomic DNA was isolated from peripheral blood leukocytes using the QIAamp DNA Mini kit (Qiagen, Hilden, Germany), following the manufacturer's protocol. Genotype determination of the SNP rs16754, a synonymous SNP within exon 7, was performed using the TaqMan SNP genotyping system (Applied Biosystems, Foster City, CA, USA) (Table I). The details of this method were previously reported (23). The assay mixes,
Table I. Primers and TaqMan probe used for Wilms' tumor 1 gene rs16754 genotyping.

\begin{tabular}{ll}
\hline Primers & \multicolumn{1}{c}{ Sequence } \\
\hline Forward & GCC TCC CTT CCT CTT ACT CTC T \\
Reverse & GATG CCG ACC GTA CAA GAG T \\
Reporter-1 (T) & VIC-CAC ACG TCG CAC ATC-NFQ \\
Reporter-1 (G) & FAM-CAC GCC GCA CAT C-NFQ \\
\hline
\end{tabular}

NFQ; non-fluorescence quencher; FAM, carboxyfluorescein.

including unlabeled polymerase chain reaction (PCR) primers, carboxyfluorescein (FAM) and VIC dye-labeled TaqMan minor groove binder probes of the Assays-by-Design system, were designed by Applied Biosystems. The reaction system contained 50 ng genomic DNA, $5 \mu 12 \mathrm{X}$ TaqMan ${ }^{\mathrm{TM}}$ Genotyping Master Mix, $0.25 \mu \mathrm{l}$ 40X Assay Mix, adjusted with Milli-Q $\mathrm{H}_{2} \mathrm{O}$ to reach a total volume of $10 \mu \mathrm{l}$. The reaction conditions included an initial step at $95^{\circ} \mathrm{C}$ for $10 \mathrm{~min}$, followed by 40 cycles at $95^{\circ} \mathrm{C}$ for $15 \mathrm{sec}$, and at $60^{\circ} \mathrm{C}$ for $60 \mathrm{sec}$ in a 96 -well plate including negative (no DNA) and positive controls to ensure genotyping accuracy using Prism ${ }^{\circledR} 7500$ Fast Real-Time PCR and GeneAmp ${ }^{\circledR}$ PCR system 7500 (both from Applied Biosystems). The genotyping results were analyzed using the Applied Biosystems 7500 software, version 2.0.5, and randomly selected to be confirmed by direct sequencing. Quality control was set at a call rate of $>95 \%$ and an accuracy rate of $>99 \%$.

Study of WT1 expression at the mRNA and protein level. Our study of WT1 mRNA expression among the rs16754 genotypes used reverse transcription quantitative PCR (RT-qPCR). RNA was extracted from 66 frozen tumor tissue samples (31 GG, $20 \mathrm{GA}$ and $15 \mathrm{AA}$ ) and 59 normal tissue samples (23 GG, 20 AG and 6 AA) with known rs16754 genotypes. RT-qPCR was performed for the WT1 gene with GAPDH as an internal control using the TaqMan probe technique. The primers and probes used in our study were previously described (24). A relative expression of the $W T 1$ gene was calculated as $\log _{10}$ (copy number of WT1/copy number of GAPDH).

The study of WT1 protein expression and localization on tumor tissue used a WT1 immunohistochemical staining method, as described in our previous publication (11). Briefly, formalin-fixed, paraffin-embedded tissue samples from CRC patients were stained overnight at $4^{\circ} \mathrm{C}$ with a mouse monoclonal anti-WT1 antibody (cat. no. clone 6FH2; Dako, Carpinteria, CA, USA), which was used as the primary antibody at a dilution of 1:100. The WT1 protein was detected by the EnVision ${ }^{\mathrm{TM}}+$ system (Dako) according to the manufacturer's instructions. Finally, the tissue sections were incubated with 3,3'-diaminobenzidine (Sigma, St. Louis, MO, USA) until a brown color developed, and then counterstained with Harris' modified hematoxylin. In negative controls, the primary antibodies were omitted. For evaluation of WT1 expression, slides of cancer specimens were analyzed by a pathologist specialised in gastroenterology (S.K.), who was blinded to the clinical information and genotyping results. To grade the tissue expression of WT1, we used the Allred scoring system, which is the standard system used to 
Table II. Allele and genotype distribution of Wilms' tumor 1 gene single-nucleotide polymorphism rs16754 in the CRC and age-matched control groups.

\begin{tabular}{lccc}
\hline & \multicolumn{2}{c}{ Subject group } & \\
\cline { 2 - 3 } Variables & CRC cases & Controls & P-value \\
\hline Total no. & 104 & 208 & \\
Mean age (range), & $51.0(16-63)$ & $51.0(17-63)$ & 0.92 \\
years & & & \\
Allelotype & & & \\
(alleles, \%) & & & \\
G & $126(60.6)$ & $289(69.5)$ & \\
A & $82(39.4)$ & $127(30.5)$ & \\
Genotype & & & \\
(cases, \%) & & & \\
GG & & & \\
GA & $41(39.4)$ & $98(47.1)$ & \\
AA & $44(42.3)$ & $93(44.7)$ & \\
Minor allele & $19(18.3)$ & $17(8.2)$ & \\
genotype (cases, \%) & & & \\
GG/GA & & & \\
AA & $85(81.7)$ & $191(91.8)$ & \\
\hline
\end{tabular}

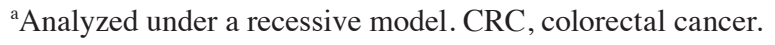

evaluate staining intensity and staining pattern. The numerical value for overall intensity (intensity score) is based on a 4-point system: 0, 1, 2 and 3 (for none, light, medium, or dark staining, respectively), while the numerical value for percentage of stained cells (proportion score) is determined by a geometric rather than linear division; no cells stained $=0 ; \leq 1 / 100$ cells stained $=1$; $\leq 1 / 10$ cells stained $=2 ; \leq 1 / 3$ cells stained $=3, \leq 2 / 3$ cells stained $=4$; and all cells stained $=5$. The sum of the two values yields the total Allred's score, which ranges between 0 and 8 .

Statistical analysis. Conformation to the Hardy-Weinberg equilibrium for rs16754 was tested by its formula. A statistical analysis of the association between the genotype frequency of each SNP and the occurrence of disease was performed using the Chi-square test. Odds ratios were calculated using univariate logistic regression analysis. A survival analysis was performed using the log-rank test. Evidence of disease progression, new metastases, second primary disease and recurrence were defined as 'progression' in the progression-free survival (PFS) analysis, while cancer-related deaths were considered as failures in the overall survival (OS) analysis. Unless otherwise stated, $\mathrm{P}<0.05$ was considered to indicate a statistically significant difference. All the statistical analyses used the statistical package Stata Release 13 (StataCorp LP, College Station, TX, USA).

\section{Results}

Clinicopathological characteristics. A total of 104 CRC cases, 41 women and 63 men, with a mean age of 51 years (range, 16-63 years) were included in this study. A total of
Table III. Clinical and pathological parameters of colorectal cancer patients and their correlation with the Wilms' tumor 1 gene (WT1) rs16754 genotype.

\begin{tabular}{|c|c|c|c|}
\hline \multirow[b]{2}{*}{ Parameters } & \multicolumn{2}{|c|}{ WT1 rs16754 genotype } & \multirow[b]{2}{*}{ P-value } \\
\hline & GG/GA $(\%)$ & $\mathrm{AA}(\%)$ & \\
\hline All $(n=104)$ & $85(81.7)$ & $19(18.3)$ & \\
\hline Gender & & & 0.20 \\
\hline Female & $36(87.8)$ & $5(12.2)$ & \\
\hline Male & $49(77.9)$ & $14(22.2)$ & \\
\hline Tumor location & & & 0.07 \\
\hline Left colon/rectum & $76(84.4)$ & $14(15.6)$ & \\
\hline Right colon & $9(64.3)$ & $5(35.7)$ & \\
\hline Tumor size, cm (range) & $5.6(2.5-16.5)$ & $5.5(2.5-12)$ & 0.82 \\
\hline Age, years & & & 0.52 \\
\hline$<40$ & $14(87.5)$ & $2(12.5)$ & \\
\hline$>40$ & $71(80.7)$ & $17(19.3)$ & \\
\hline T-stage & & & 0.90 \\
\hline $1-2$ & $8(80.0)$ & $2(20.0)$ & \\
\hline $3-4$ & $76(81.7)$ & $17(18.3)$ & \\
\hline N stage & & & 0.51 \\
\hline No & $30(79.0)$ & $8(21.0)$ & \\
\hline N1-2 & $53(84.1)$ & $10(15.9)$ & \\
\hline M stage & & & 0.97 \\
\hline M0 & $66(81.5)$ & $15(18.5)$ & \\
\hline M1 & $18(81.8)$ & $4(18.2)$ & \\
\hline AJCC stage & & & 0.60 \\
\hline $0-2$ & $30(79.0)$ & $8(21.0)$ & \\
\hline $3-4$ & $54(83.1)$ & $11(16.9)$ & \\
\hline CEA, ng/dl & & & 0.31 \\
\hline$<5$ & $49(86.0)$ & $8(14.0)$ & \\
\hline$>5$ & $36(78.3)$ & $10(21.7)$ & \\
\hline Differentiation & & & 0.96 \\
\hline High & $45(81.8)$ & $10(18.2)$ & \\
\hline Moderate/poor & $35(81.4)$ & $8(18.6)$ & \\
\hline $\begin{array}{l}\text { Lymphovascular/ } \\
\text { perineural invasion }\end{array}$ & & & 0.18 \\
\hline No & $58(85.3)$ & $10(14.4)$ & \\
\hline Yes & $23(74.2)$ & $8(25.8)$ & \\
\hline Lymph node ratio & & & 0.07 \\
\hline$<0.35$ & $62(86.1)$ & $10(13.9)$ & \\
\hline$>0.35$ & $19(70.4)$ & $8(29.6)$ & \\
\hline Survival analysis ${ }^{\mathrm{a}}, \%$ & & & $<0.01$ \\
\hline 3-year PFS & 67.00 & 42.90 & \\
\hline 3-year OS & 80.60 & 59.20 & \\
\hline
\end{tabular}

${ }^{a}$ Log-rank test. CEA, carcinoembryonic antigen; PFS, progression-free survival; OS, overall survival; AJCC, American Joint Committee on Cancer.

208 controls were used (67 men and 141 women), with a mean age of 51 years (range, 17-63 years), all of whom resided in the same geographic area as the patients (Table II). 


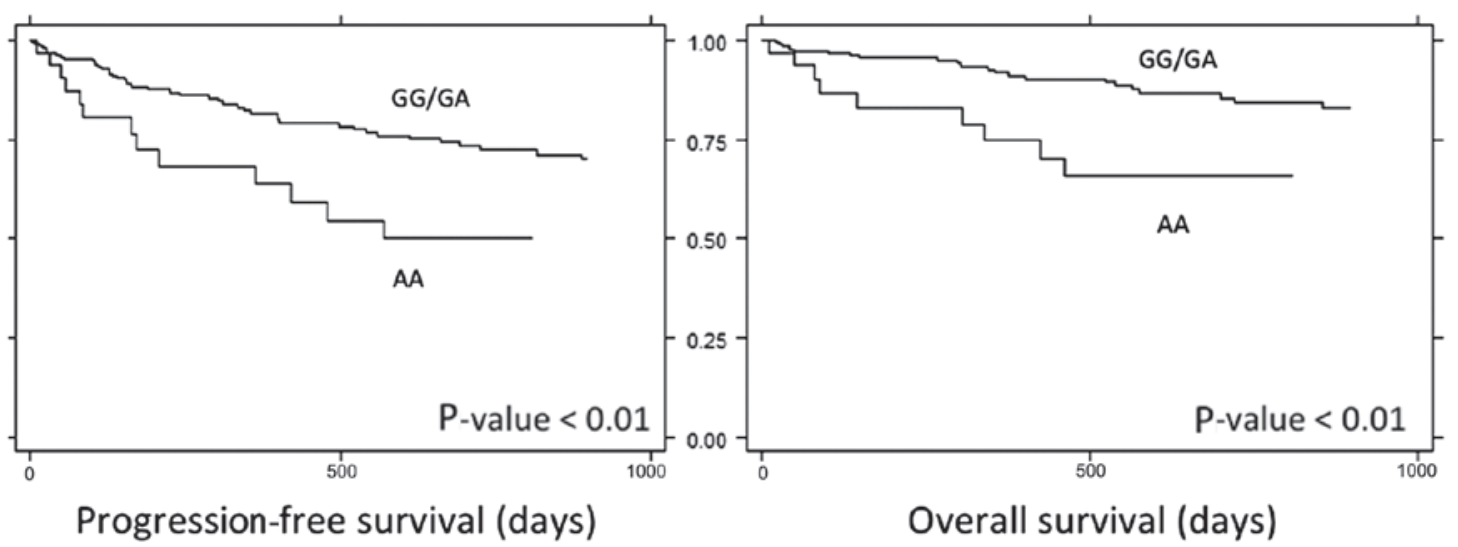

Figure 1. Kaplan-Meier curves showing the differences in progression-free and overall survival between colorectal cancer cases with different genotype groups of Wilms' tumor 1 gene rs16754 polymorphism.

A

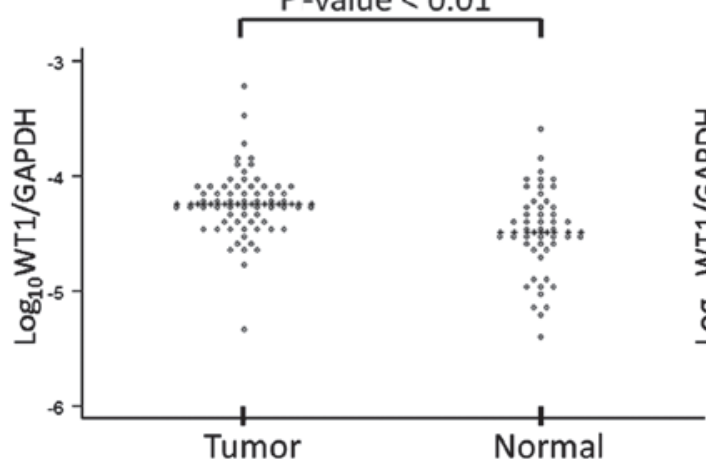

B

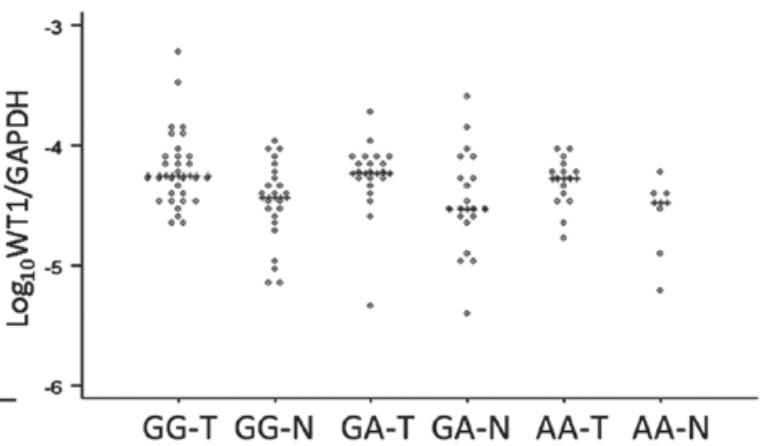

Figure 2. Relative expression of Wilms' tumor 1 gene (WT1) mRNA as revealed by quantitative polymerase chain reaction analysis. (A) Comparison between tumor and normal tissue and (B) normal tissue comparison of different genotype groups.

Genotyping by the TaqMan-SNP genotyping assay yielded a call rate of $>99 \%$ and a reproducibility (accuracy) rate of $100 \%$. The genotype distribution conformed to the Hardy-Weinberg equilibrium $(\mathrm{P}=0.80)$. The minor allele frequency (MAF) of the rs16754 (allele A) was 0.33. The MAF among CRC cases (0.39) was significantly higher compared with that in controls $(0.31)(\mathrm{P}=0.03)$. The AA genotype was significantly associated with CRC (odds ratio $=2.51$, 95\% confidence interval: 1.24-5.07, $\mathrm{P}=0.01$ ).

The WT1 genotype groups were not significantly associated with any clinicopathological parameters in the CRC cases (Table III). The mean follow-up period was 34 months (range, 6-90 months). When the genotypes were analyzed against survival, it was found that the AA genotype was associated with poorer PFS and OS (Fig. 1). The 3-year PFS in the AA genotype group was $43 \%$, compared with $67 \%$ in other genotypes $(\mathrm{P}<0.01)$. Furthermore, the 3 -year $\mathrm{OS}$ in the AA genotype group was $60 \%$, compared to $81 \%$ in other genotypes $(\mathrm{P}<0.01)$.

On RT-qPCR, the relative expression of $W T 1$ in the tumor tissue was significantly higher compared with that in the adjacent normal colonic mucosa $(\mathrm{P}<0.01)$. However, there were no significant differences in the relative expressions among the three genotype groups (Fig. 2). On immunohistochemical staining in the 101 cases for which histological slides were obtained, the majority of CRC tumors (94 cases, 93\%) exhibited positive staining results. Apart from one case in the AA group, which displayed nuclear staining, all immunoreactivity was localized in the cytoplasm. The average Allred scores in the AA (6.4) and GA (6.7) genotype groups were higher compared with those in the GG group (5.9); however, the differences did not reach statistically significant levels $(\mathrm{P}=0.09)$.

\section{Discussion}

The oncologic role of $W T 1$ has been validated in various types of cancer, including CRC (13-16). Our previous study demonstrated a correlation between high expression of the WT1 protein and poor outcome in CRC (11). In the present study, we focused on a genetic variant of WT1 and its association with this disease. The rs16754 SNP, located in exon 7 of WT1, has been found to exhibit a significant correlation between its major allele and relatively favorable clinical outcomes in hematological malignancies (18-20). According to the 1000 Genomes Browsers (browser.1000genomes.org) (25), A is the major allele of rs16754 in Western populations, while $\mathrm{G}$ is the major allele in East Asian populations $(26,27)$. The MAF of 0.31 in our study was comparable with previous reports from other Asian populations in the 1000 Genomes database and in reverse correlation to the MAFs reported in European populations (18). 
The CRC patients included were limited to those aged $<65$ years, in order to exclude potential factors associated with ageing that may affect disease development. In addition, there was a certain difficulty in recruiting older age-matched controls in our community. Our analysis demonstrated that the MAF of the rs16754 in CRC cases significantly deviated from that in the age-matched controls. The subsequent genotype analysis demonstrated a significant association between the AA genotype and CRC prognosis. As the WT1 protein expression has been identified as an unfavorable prognostic marker in CRC (11), it is possible that this variant exhibits a correlation with expression levels. Our finding of WT1 overexpression in CRC tissue was consistent with previous reports $(11,16)$. Although differences in WTI expression in blood leukocytes among rs16754 genotypes have been reported in a previous study (18), a recent study on pediatric leukemia failed to detect any significant association between expression and genotype (28). Consistent with the latter study, our findings suggest that the expression level of WT1 in colonic tissue is independent of the rs16754 genotype. As the rs16754 is a synonymous SNP, the association may not indicate a direct causal association between the gene and disease development. However, the SNP may be in a linkage relationship with other disease-causal variants of WTI.

In conclusion, our study identified a genetic association of the rs16754 of WT1 in a group of Thai CRC patients aged $<65$ years. The study also demonstrated a significant correlation between risk genotype (AA) and poorer outcome following multimodal treatment. The data suggest that this variant may be a candidate disease marker in CRC.

\section{Acknowledgements}

This study was supported by the Thailand Research Fund (grant no. MRG5208118). Surasak Sangkhathat is supported by the Anandamahidol Foundation. Dave Patterson reviewed the English in the manuscript.

\section{References}

1. Jemal A, Center MM, DeSantis C and Ward EM: Global patterns of cancer incidence and mortality rates and trends. Cancer Epidemiol Biomarkers Prev 19: 1893-1907, 2010.

2. Khuhaprema T and Srivatanakul P: Colon and rectum cancer in Thailand: An overview. Jpn J Clin Oncol 38: 237-243, 2008.

3. Khuhaprema T, Attasara P, Sriplung H, Wiangnon S and Sangrajrang S (eds). Cancer in Thailand. Vol 7. National Cancer Institute, Bangkok, Thailand, pp2007-2009, 2013.

4. Sriplung $\mathrm{H}$, Bilheem S, Kuntipundee $\mathrm{T}$ and Geater SL: Differences in cancer incidence among predominantly Muslim and Buddhist subpopulations in Songkhla. Asian Pac J Cancer Prev 15: 9979-9983, 2014.

5. Kritsanasakul A, Boonpipattanapong T, Wanitsuwan W, Phukaoloun M, Prechawittayakul P and Sangkhathat S: Impact of lymph node retrieval on surgical outcomes in colorectal cancers. J Surg Oncol 106: 238-242, 2012.

6. Boonpipattanapong T and Chewatanakornkul S: Preoperative carcinoembryonic antigen and albumin in predicting survival in patients with colon and rectal carcinomas. J Clin Gastroenterol 40: 592-595, 2006.

7. Liu Z, Zhang Y, Niu Y, Li K, Liu X, Chen H and Gao C: A systematic review and meta-analysis of diagnostic and prognostic serum biomarkers of colorectal cancer. PLoS One 9: e103910, 2014.

8. Coppedè F, Lopomo A, Spisni R and Migliore L: Genetic and epigenetic biomarkers for diagnosis, prognosis and treatment of colorectal cancer. World J Gastroenterol 20: 943-956, 2014.
9. Belov L, Zhou J and Christopherson RI: Cell surface markers in colorectal cancer prognosis. Int J Mol Sci 12: 78-113, 2010.

10. Chaiyapan W, Duangpakdee P, Boonpipattanapong T, Kanngern S and Sangkhathat S: Somatic mutations of K-ras and BRAF in Thai colorectal cancer and their prognostic value. Asian Pac J Cancer Prev 14: 329-332, 2013.

11. Bejrananda T, Phukaoloun M, Boonpipattanapong T, Wanitsuwan W, Kanngern S, Sangthong R and Sangkhathat S: WT1 expression as an independent marker of poor prognosis in colorectal cancers. Cancer Biomark 8: 35-42, 2010-2011-2011.

12. Cheever MA, Allison JP, Ferris AS, Finn OJ, Hastings BM, Hecht TT, Mellman I, Prindiville SA, Viner JL, Weiner LM, et al: The prioritization of cancer antigens: A National Cancer Institute pilot project for the acceleration of translational research. Clin Cancer Res 15: 5323-5337, 2009.

13. Hu SY, Gu WY, Chen ZX, Wang XL, Cen JN, He HL, Chai YH and Chen CS: The significance of detecting WT1 expression in childhood acute leukemias. Pediatr Hematol Oncol 27: 581-591, 2010.

14. Oji Y, Miyoshi S, Maeda H, Hayashi S, Tamaki H, Nakatsuka S, Yao M, Takahashi E, Nakano Y, Hirabayashi $\mathrm{H}$, et al: Overexpression of the Wilms' tumor gene WT1 in de novo lung cancers. Int J Cancer 100: 297-303, 2002.

15. Domfeh AB, Carley AL, Striebel JM, Karabakhtsian RG, Florea AV, McManus K, Beriwal S and Bhargava R: WT1 immunoreactivity in breast carcinoma: Selective expression in pure and mixed mucinous subtypes. Mod Pathol 21: 1217-1223, 2008.

16. Oji $Y$, Yamamoto $H$, Nomura $M$, Nakano $Y$, Ikeba A, Nakatsuka S, Abeno S, Kiyotoh E, Jomgeow T, Sekimoto M, et al: Overexpression of the Wilms' tumor gene WT1 in colorectal adenocarcinoma. Cancer Sci 94: 712-717, 2003.

17. Oka Y and Sugiyama H: WT1 peptide vaccine, one of the most promising cancer vaccines: Its present status and the future prospects. Immunotherapy 2: 591-594, 2010.

18. Damm F, Heuser M, Morgan M, Yun H, Grosshennig A, Göhring G, Schlegelberger B, Döhner K, Ottmann O, Lübbert $\mathrm{M}$, et al: Single nucleotide polymorphism in the mutational hotspot of WT1 predicts a favorable outcome in patients with cytogenetically normal acute myeloid leukemia. J Clin Oncol 28: 578-585, 2010.

19. Becker H, Maharry K, Radmacher MD, Mrózek K, Metzeler KH, Whitman SP, Schwind S, Kohlschmidt J, Wu YZ, Powell BL, et al: Clinical outcome and gene- and microRNA-expression profiling according to the Wilms tumor 1 (WT1) single nucleotide polymorphism rs16754 in adult de novo cytogenetically normal acute myeloid leukemia: A Cancer and Leukemia Group B study. Haematologica 96: 1488-1495, 2011.

20. Chen X, Yang Y, Huang Y, Tan J, Chen Y, Yang J, Dou H, Zou L, Yu J and Bao L: WT1 mutations and single nucleotide polymorphism rs16754 analysis of patients with pediatric acute myeloid leukemia in a Chinese population. Leuk Lymphoma 53: 2195-2204, 2012.

21. Chalieopanyarwong V, Boonpipattanapong T, PrechawittayakulP and Sangkhathat S: Endoscopic obstruction is associated with higher risk of acute events requiring emergency operation in colorectal cancer patients. World J Emerg Surg 8: 34, 2013.

22. Compton CC and Greene FL: The staging of colorectal cancer: 2004 and beyond. CA Cancer J Clin 54: 295-308, 2004.

23. Phusantisampan T, Sangkhathat S, Phongdara A, Chiengkriwate $\mathrm{P}$, Patrapinyokul S and Mahasirimongkol S: Association of genetic polymorphisms in the RET-protooncogene and NRG1 with Hirschsprung disease in Thai patients. J Hum Genet 57: 286-293, 2012.

24. Sangkhathat S, Kanngurn S, Chaiyapan W, Gridist P and Maneechay W: Wilms' tumor 1 gene (WT1) is overexpressed and provides an oncogenic function in pediatric nephroblastomas harboring the wild-type WT1. Oncol Lett 1: 615-619, 2010.

25. Abecasis GR, Altshuler D, Auton A, Brooks LD, Durbin RM, Gibbs RA, Hurles ME and McVean GA; 1000 Genomes Project Consortium: A map of human genome variation from population-scale sequencing. Nature 467: 1061-1073, 2010.

26. Altshuler DM, Gibbs RA, Peltonen L, et al; International HapMap 3 Consortium: Integrating common and rare genetic variation in diverse human populations. Nature 467: 52-58, 2010.

27. Lauhakirti D, Sritana N, Boonthimat C, Promsuwicha O and Auewarakul CU: WT1 mutations and polymorphisms in Southeast Asian acute myeloid leukemia. Exp Mol Pathol 91: 682-686, 2011.

28. Ho PA, Alonzo TA, Gerbing RB, Kuhn J, Pollard JA, Hirsch B, Raimondi SC, Gamis AS and Meshinchi S: The prognostic effect of high diagnostic WT1 gene expression in pediatric AML depends on WT1 SNP rs16754 status: Report from the Children's Oncology Group. Pediatr Blood Cancer 61: 81-88, 2014. 